\title{
Experimental Model for Studying the Prefrontal EEG Biomarkers in Correlation with Food Intake Behavior: Neurofeedback Platform
}

\author{
Mohammed I. Al-Hiyali ${ }^{1}$, Asnor J. Ishak ${ }^{1,2}$, Hafiz Harun1, Siti A. Ahmad ${ }^{1,2}$, and Wan Sulaiman, W. A . $^{2,3}$. \\ ${ }^{1}$ Control System \& Signal Processing Research Centre, Department of Electrical and Electronic Engineering, University Putra \\ Malaysia, 43400 UPM Serdang, Selangor, Malaysia \\ ${ }^{2}$ Malaysian Research Institute on Ageing, University Putra Malaysia, 43400 UPM Serdang, Selangor, Malaysia \\ ${ }^{3}$ Faculty of Medicine and Health Sciences, University Putra Malaysia, 43400 UPM Serdang, Selangor, Malaysia. \\ Corresponding author: Asnor J. Ishak (e-mail: asnorji@upm.edu.my).
}

This research is financially supported by a Putra Grant from University Putra Malaysia under Grant 9606000.

\begin{abstract}
Background: This study aims to investigate the effects of visual neurofeedback stimulation on the brain activity in overweight cases. The neuroscience studies indicated the personal decision about eating under the impact of environmental factors such as (visually, smelling, tasting) is related to neural activity of the prefrontal lobe of the brain. Therefore, there were many attempts to modify the food intake behavior in overweight cases through the stimulation of the prefrontal cortex. However, the empirical viewing of EEGneurofeedback experiments has not explicated the details about the effect of the EEG-NF, the specificity of positive treatment effects remains in a challenging scope.
\end{abstract}

Methods: This study is a cue-exposure EEG-NF experiment to verify the hypothesis of effecting the EEG-NF on the electrical activity of PFC and modifying the general symptoms of food intake behavior in overweight cases. Twenty-four individuals were recruited as participants for this study. These participants were assigned randomly into two groups; the EX-Group $(\mathrm{N}=12)$ enrolled in 8 sessions of the EEG-NF experiment, and the C-Group ( $\mathrm{N}=12)$ was listed in no EEG-NF sessions. The participants provided researchers with a self-report questionnaire relating to their observation of general symptoms of food intake behavior, and EEG signals recordings into the pre and posts stimulation phase. The power spectral density (PSD) method was applied for EEG parameters extraction. 
Results: The results of a two-way analysis of variance (ANOVA) explained that a significant variation in variables between the two groups after the EEG-NF experiment. The analysis of the quantitative variables indicated that the effect of EEG-NF experiment was a significant decrement in EEG power bands which significantly influenced changing the median of self-report questionnaire responses that is related to general symptoms of food intake behavior.

Conclusions: This study provides preliminary support for the therapeutic potential of EEG-NF experiment that targets the prefrontal cortex, to influence neural processes underlying food intake behavior in overweight cases.

Keywords: EEG-Neurofeedback, Overweight, Obesity, Signal Processing, Power Spectral Density

\section{Background}

The World Health Organization (WHO) reported in 2016, more than 1.9 billion adults are overweight and more than 650 million are obese [1]. These individuals are not capable of regulating their food intake behavior, which is considered key to weight gain. Food Intake (FI) regulation is a complex process involving the combination of internal factors such as neural signals, as well as external factors including the environmental factors that stimulate eating desire such as sight, smell, and taste [2]. However, studies have shown that specific areas of the brain are involved in the interactive processing of food vs. non-foodrelated visual stimuli in the different states of hunger and satiety, these include the PreFrontal Cortex (PFC). Another study shows that food, even when presented only as an image, will cause a larger hunger response in evolutionarily conserved brain areas, sustaining survival, in particular, because the visual presentation of the food was possibly the first way of food contact [3]. The recent progress in brain activity research found a therapeutic program that targeted stimulation in the decision-making process may lead to an encouraging approach in the prevention of weight gain.

The studies of brain-direct stimulation in overweight and obesity individuals have previously shown to have an impact on food intake behavior. Since the frontal lobe is involved in decision-making and the process of cognitive controls, the PFC stimulation has been suggested by many researchers to improve the food intake behaviors in overweight and obesity [4], [5]. The common non-invasive techniques, such as the Transcranial Magnetic Stimulation (TMS), transcranial Direct Current Stimulation (tDCS) have been applied to stimulate PFC for those cases [6], [7]. According to the findings of [8], systematic review in tDCS appears 
to be effective in modifying food intake behavior, the PFC stimulation has a significant impact on the treatment of the cognitive deficit which is related to food intake behavior. However, these findings may be somehow limited in the assessment methods. The experiment's efficiency had been assessed mostly based on qualitative variables and rarely focused on quantitative variables. The qualitative variables were extracted from the behavior report in two phases, namely pre, and post-stimulation to verify the difference in reports, while the quantitative variables were extracted from neurocognitive functions.

For the importance of quantitative analysis in understanding the experiment performance trends and examining the neurocognitive functions through which PFC stimulation affects food intake behaviors, some studies such as [9] combined two devices, stimulation device, and EEG data acquisition device for collecting the raw EEG signals. However, the experiment procedures using two devices takes extra time to replace the electrodes and supported by the extra budget.

Neurofeedback is one of the brain-stimulation techniques that involved a real-time neuro-signal measurement, immediate data processing with the extraction of the neurophysiology parameters and feedback to individuals to make changes in brain functioning and consequently behaviors, this technique classified to FMRI-NF and EEG-NF based on neuroimaging data acquisition [10], that means the NF experiment setup does not need to external data acquisition for quantitative variables extraction.

However, the experiment works in the literature of NF used the FMRI-NF device to PFC stimulation in excess weight individuals to change food intake behavior [11], [12], while the EEG-NF has not yet been applied to PFC stimulation in these cases, although the EEG device is affordable and easy handle compared with FMRI. Also, the total number of EEG-NF studies in the eating behavior area are very scares compared with other techniques [13]. Therefore, this study hypothesizes that EEGneurofeedback stimulating the prefrontal activity and leads to modifying the general symptoms of food intake behaviors in experiment participants.

To prove or deny this hypothesis, there are two research questions; what is the quantitative difference in EEG power between pre and post-NF stimulation sessions; and what is the qualitative difference in self-report behavior between pre and poststimulation sessions. The EEG signals have highly random characteristics and contain useful data about the mental state of cases of study. The EEG signals characteristics are non-linear and non-stationary, hence, the important data can be extracted by using signal processing techniques. 
The EEG signals included brain waves divided into a set of frequency bands, these bands from low and high frequencies are; Delta, Theta, Alpha, and Beta [22]. Frequency indicates the speed of a wave's oscillation which is measured by the number of waves per second and measured in Hertz $(\mathrm{Hz})$. The activity in these frequency bands is related to the brain states and functions. The delta band mostly dominates deeper sleep states. Theta band is associated with drowsiness and early stages of sleep and has also been shown as a mechanism for cognitive control in the frontal brain cortex [33]. The alpha band is correlated with an awake or relaxed state. Most individuals begin to increase alpha band in less than half a minute when he or she simply closes his or her eyes and thinks of something peaceful. The alpha band is typically observed in the occipital and posterior regions of the brain [34]. The beta band has been related to concentration, and state of intellectual activity [35]. Both of beta and alpha bands are referred to as fast oscillation bands while theta and delta bands are referred to as slow oscillation bands. The literature on fundamentals of EEG signals and brain activity has documented when an excessive amount of slow oscillation bands or insufficient amount of fast oscillation bands occurs in the frontal brain lobe, it becomes difficult for the individual to control his or her behavior, attention, and/or emotions. This inefficient pattern of activity might lead to a deficiency in concentration, memory, intellectual efficiency, and be controlling mood [36]. Neuroscientific studies have reported that cognitive processes are related to EEG oscillation. Hence, the regulation of EEG oscillation should have an impact on modifying the subject's behavior [37].

The main objective of this study is to quantitatively investigate the effects of EEG-Neurofeedback stimulation on the EEG power bands in PFC. The sub-objectives to validate this experiment are; Extract the quantitative and qualitative variables, the quantitative variables are extracted from EEG signals, and qualitative variables are obtained from the self-behavioral report, to verify the variance in experiment variables between groups at the pre and post-stimulation phases, and to find the correlation between them in two study phases.

\section{Methods}

The study design is the Randomized Control Trial (RCT) to recruit the participants. Two groups are participating, EX-group and C-groups with two conditions (pre and post-stimulation) the study structure is shown in Appendix A (Figure 1). The EEG signal and self-report questionnaire are included in data collection procedures. The EEG data is recorded utilizing 2 channels clinical system: Plus, BrainAvatar software. This system and the software are specified for EEG systems and neurofeedback stimulation. All EEG signals that were collected throughout the sessions are processed to spectrum estimation for EEG parameters extraction, and food intake assessment was done by analysing the self-report questionnaire terms. All 
data are analysed and examined for variance at pre and post-stimulation, an overview of the experiment setup shown in Appendix A (Figure 2).

\section{Participants}

The ideal number of participants to be involved in this study is calculated by using the Hypothesis testing method [14]. The null hypothesis defined is that food intake behaviour is not different among groups. The alternative hypothesis defined is that food intake behaviour is significantly different among groups. Consequently, the food intake behaviour is selected as the test variable and the impact of EEG-NF stimulation on this behaviour has been extracted from a previous study [15], as shown in appendix B (Participants Size Calculation). Therefore, the suitable target number of participants per group is 12 or more. However, in this study, the total number of participants recruited is 24 .

Regarding the study location, the study was performed at the Clinical Neurophysiology Clinic at Medical Lab-Faculty of Medicine and Health Sciences, University Putra Malaysia. Participants were recruited from University Putra Malaysia (UPM) through advertisements distributed by student on social media groups, advertisement boards at libraries, and the main entrance of faculties. The potential participants are from different races including local and international students who were asked to undergo a screening test to assess their eligibility according to the following criteria. The Inclusion criteria; Participants must have body mass index more than or equal $25 \mathrm{~kg} / \mathrm{m}^{2}$, Participants must be within the defined age limit (18 45) years, Participants provide written consent, Participants must not have suffered any head injury or brain disorders. While the exclusion criteria; Failed to meet the inclusion criteria demands, currently undergoing treatment with drugs evoking weight variation, currently pregnant, currently smoking, failed to provide written consent. The enrolment process took one month, where thirty respondents were assessed for acceptability. Twenty-four respondents managed to fulfil the inclusion criteria while six respondents were excluded. After receiving information about the aims of the study, each participant provided a written consent form to participate in the study.

\section{EEG Recording \& Neurofeedback Stimulation}

The EEG signal is recorded, and NF applied by Atlantis device is manufactured by the BrainMaster company. Brain-Master is one of the standard programs for applying neurofeedback and hosts a wealth of research articles that have been used in its development. The device has been calibrated and tested in the manufacturer's facility and ready to use for the collection of EEG data and NF stimulation. EEG signals were collected using two surface gold electrodes to measure the brain signal on the scalp with ear-clip electrodes measuring the reference signals. The EEG-NF device aims to stimulate the PFC by using 
the protocol in visual and auditory stimulation modality. Since the target brain area of stimulation is PFC, the electrodes were placed on prefrontal according to $10 / 20$ international system, and the head-size was measured for participants to identify these positions, the electrodes positioning steps illustrated in Figure 1.

The bipolar montage was used, the active electrodes were placed on the left and right prefrontal positions (Fp1-Fp2); the reference electrodes were placed on the left and right (A1-A2) earlobe as shown in Figure 2. This montage was used to record the potential voltage of brain signal between Fp1-A1 recorded in channel 1 which was marked in green colour, while Fp2-A2 recorded in channel 2 was marked in red colour. The participants are seated on a comfortable chair facing the client screen. Then, electrodes are placed on their forehead and earlobes after skin preparation. The touching area is cleaned by using a skin preparation gel which acts to minimize the skin impedance while also enhancing the performance of the electrode monitoring. The conductive paste was used to stick the electrodes in place to obtain the optimal balance of adhesiveness and conductivity. After using electrodes, they are soaked in distilled water for one minute and the rest of the paste inside the electrode is removed using a soft toothbrush. 


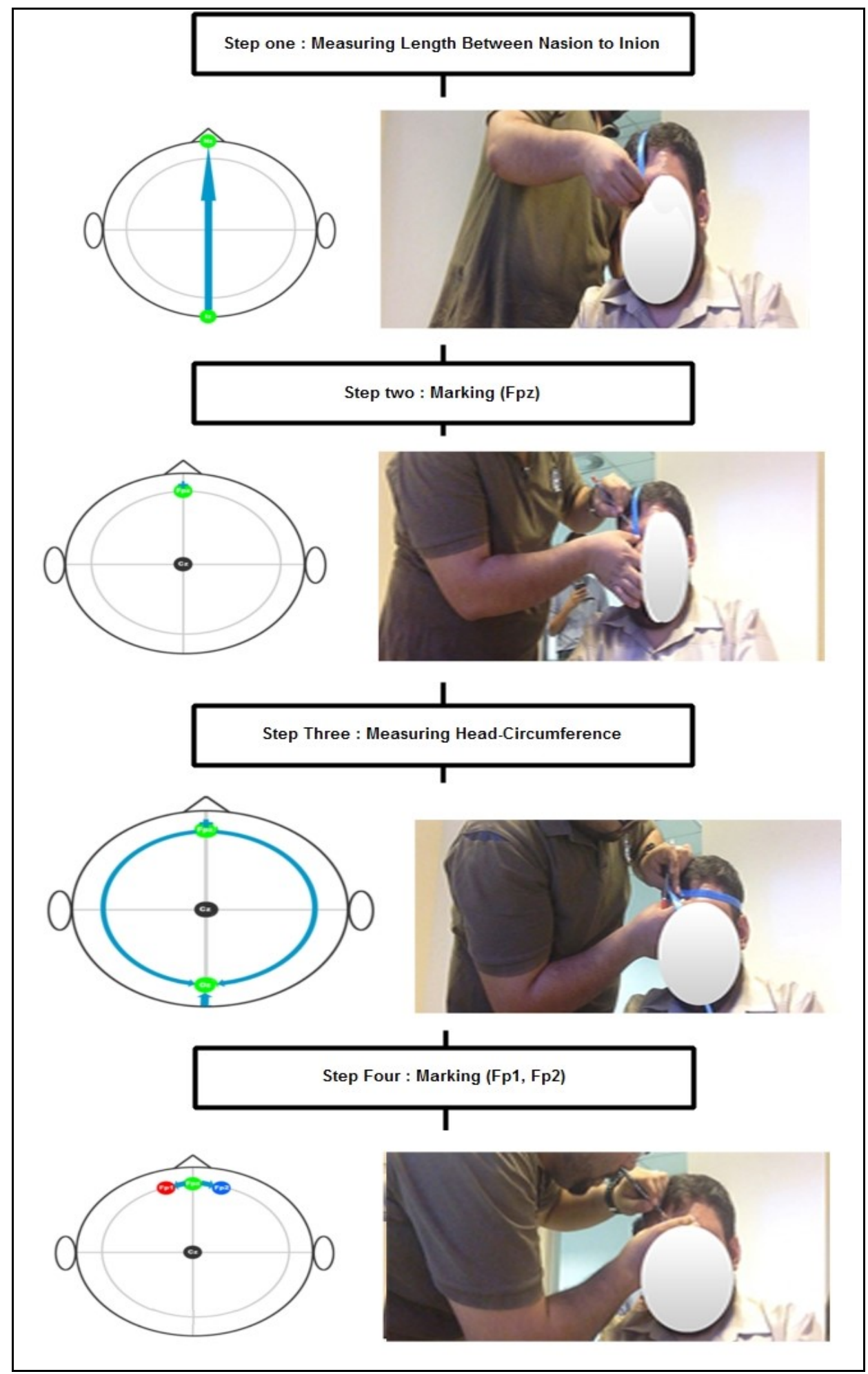

Figure 1: Electrodes Positioning 




Figure 2: Electrodes Placement

\section{Screening-Form \& Questionnaires}

The screening form was prepared for the present study to collect information about the participants; which covers sociodemographic data such as age, gender, address, email address, contact number, occupation, and emergency contact name and number. Other details consisted of weight, height, BMI, smoking term, history in mental disorders, medicine evoking weight variation, diabetic and pregnant for female.

The self-report questionnaires were designed into two main sectors to assess the food-intake behaviour. The first sector is a questionnaire (A) applied in pre-stimulation phase, it has six terms as shown in Appendix C. These terms are related with Food-Intake Behaviour (FIB), Craving Action (CA), Preoccupied with Food (PWF), Feeling, Trouble Stopping Eating (TSE), and Emotions. The tendencies of questionnaire responses have been defined as two-sides, positive and negative food intake behaviour. The positive tendency has scored 1 value, and negative behaviour has scored 3 values, while the grey area between these tendencies has scored 2 values. The FIB is scored as Normal (1), Neither Normal nor Abnormal (2), Normal (3), with other terms have a set of answers scored: Disagree (1), Neither Disagree nor Agree (2), Agree (3).

The second questionnaire sector is B, attached in Appendix C, applied in the post-stimulation phase, and has the same terms that were already included in questionnaire A with adding a section for self-report side-effect. The first three items asked general questions about appetite and evaluate how the participant was feeling during the stimulation sessions. Each item has a set of answers with the following score: 1(Excellent), 2 (Very good), 3 (Good), 4 (Average), 5 (Bad), 6 (Very bad), 7 (Terrible). 


\section{Data Collection Phases}

As mentioned before the study is RCT design with pre-post phases, all participants are randomly assigned to either the Experimental Group (EX) or Control Group (C). The EX-group is undergoing NF stimulation sessions and the C-group was not subject to be examined. The data collection included four phases; pre-session phase, NF stimulation sessions phase, postsession phase, and follow-up which aims to collect data in pre, and post-stimulation applied. The data collected during the stimulation phase is used to evaluate the changes in the EEG signal associated with stimulation. Two types of data are collected: EEG signals and self-report questionnaires.

In the first stage of the pre-stimulation phase, all participants were provided with the screening form and food intake questionnaire A. After that, all participants took an EEG recording session for 5 minutes. The participants were asked to relax with their eyes open and to focus on one of the spectral colours that were shown on the screen.

The NF Stimulation protocol is designed according to the prerequisites for good EEG-NF study that have been mentioned in the recent review [16]. Eight sessions were chosen and each session lasted for 17 min that would be convenient for research, based on the number of sessions in previous EEG-NF studies [17] [18]. These sessions are distributed toward two sessions per week. Each session is composed of 3 stimulation trials which are 4 minutes for each trial plus a 1 -minute break between them and a pre and post EEG screening as shown in Figure 3. The first 90 seconds were taken for cue exposure task while the last 90 seconds for relaxation with eyes open.

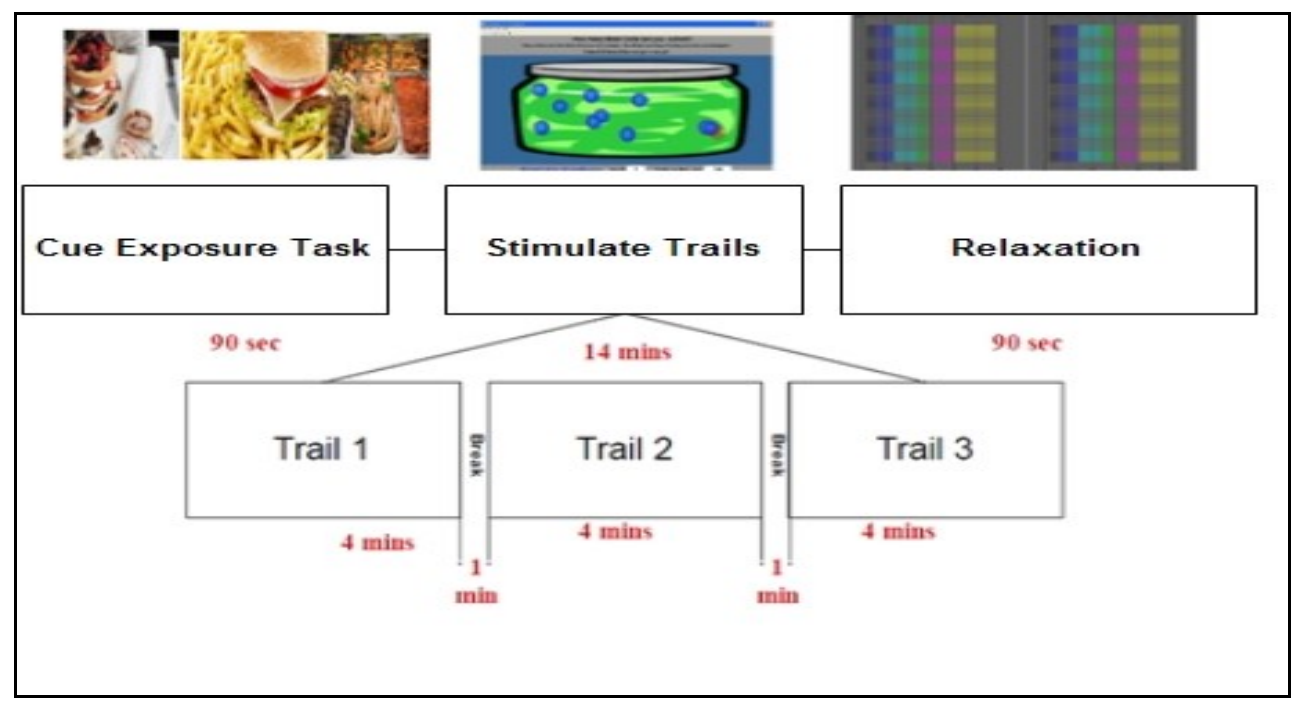

Figure 3. Stimulation Phase 
In the cue exposure task, the participants were presented with digital photographs of palatable food items such as dessert, snack food, fast-food and main dishes that are selected by the participant, and that frequently drives them to excessive food intake. During this task, the participants were instructed to focus on the photos that are shown on the screen and imagine the taste as clearly as possible. After 90 seconds, these photos were removed for following stimulation trials.

The visual feedback was performed through a flash game shown on the screen. In this part, the participant must focus on this game and try to follow the instruction as shown. After the last stimulation trials, the flash game was removed, and the participants were asked to relax with his/her eyes open for 90 seconds.

Then, after one month, all participants in both groups were called for a post-stimulation session. In this session, the participants have been asked to complete the food intake questionnaire B and perform another 5 minutes EEG recording. The Participants in EX-Group were finally administered the Food intake questionnaire A, after 2 weeks on the last session.

\section{$\underline{\text { Data Analysis }}$}

After data collection, the features were extracted from questionnaires and EEG signals were then inserted as data in SPSS (Statistical Package for Social Sciences) for statistical analysis. The statistical methods applied to the questionnaire terms to assess the food intake behaviour between pre-post stimulation phases, while the features of EEG signals have been extracted by the signal processing method. These features were then analysed by statistical methods to assess the EEG signals in the pre-post stimulation session and during the stimulation sessions.

\section{EEG signal processing}

An extension application of the BrainAvatar software was been used to export the raw EEG data and standard frequency bands of data for each session, along with the raw EEG. Next, it decomposed into several range bands, delta (1-3Hz), theta $(4-7 \mathrm{~Hz})$, alpha $(8-12 \mathrm{~Hz})$, beta $(12-30 \mathrm{~Hz})$, and gamma $(30-42 \mathrm{~Hz})$. All data collected by the EEG-NF device during the study phases were exported in the EDF file format (European Data Format). The EDF is read in MATLAB, then all data were processed with offline analyses by using the EEG toolbox in MATLAB [19]. The EEG signal processing is explained in Figure 4.

The first step is pre-processing to filter the raw data by removing artefacts data such as eye-blinking, body motion, itching or, other artefacts were sourced from an EEG equipment or the electrodes; therefore, the visual inspection and Infomax ICA (Independent Component Analysis) algorithm had applied on raw signals for filtering, the principle of this algorithm is the 
information-maximization approach to blind separation and blind deconvolution, sources are assumed independent and do not have mutual information, the information I(n) is measured by:

$$
\begin{gathered}
I(n)=\int p(X) \log \frac{1}{p(x)} d x \\
I(X, Y)=\int p(x, y) \log \frac{1}{p(x, y)} d x d y
\end{gathered}
$$

Where is a $\mathrm{p}(\mathrm{x})$ is the raw signals, $\mathrm{I}(\mathrm{n})=\mathrm{I}(\mathrm{x}), \mathrm{I}(\mathrm{y}) \ldots$ independent information, $\mathrm{I}(\mathrm{X}, \mathrm{Y})$ Joint Entropy, while mutual information $\mathrm{M}(\mathrm{X}, \mathrm{Y})$ is given by equation 3, that means the minimizing $\mathrm{M}(\mathrm{X}, \mathrm{Y})$ equal to maximizing $\mathrm{I}(\mathrm{X}, \mathrm{Y})$.

$$
M(X, Y)=I(x)+I(Y)-I(X, Y)
$$

In features extraction step, the P-welch function was applied to estimate the power spectral density of EEG signals, the data sequence $\mathrm{xi}(\mathrm{n})$ in this function can be represented as:

$$
X i(n)=x(n+i D)
$$

Here $n=0,1,2,3 \ldots . \mathrm{M}-1$, and $\mathrm{I}=0,1,2 \ldots \ldots \mathrm{L}-1$, where $\mathrm{iD}$ is the beginning of the $\mathrm{i}$ 'th sequence.

This will lead to formation of $\mathrm{L}$ data segments each of length $2 \mathrm{M}$. the modified periodogram is given as:

$$
\widetilde{P_{x x}^{i}}(f)=\left|\frac{1}{M U} \sum_{n=0}^{M-1} x(n) \omega(n) e^{-12 \pi f(n)}\right|^{2}
$$

Where $\mathrm{U}$ is the normalization factor for the power in the window function given as:

$$
U=\frac{1}{M} \sum_{n=0}^{M-1} \omega^{2}(n)
$$

Where is $\omega(n)$ is the window function. The P-Welch power spectrum which is the average of these periodogram is given as:

$$
P_{X X}^{W}(f)=\frac{1}{L} \sum_{i=0}^{L-1} \widetilde{P_{x x}^{i}}(f)
$$




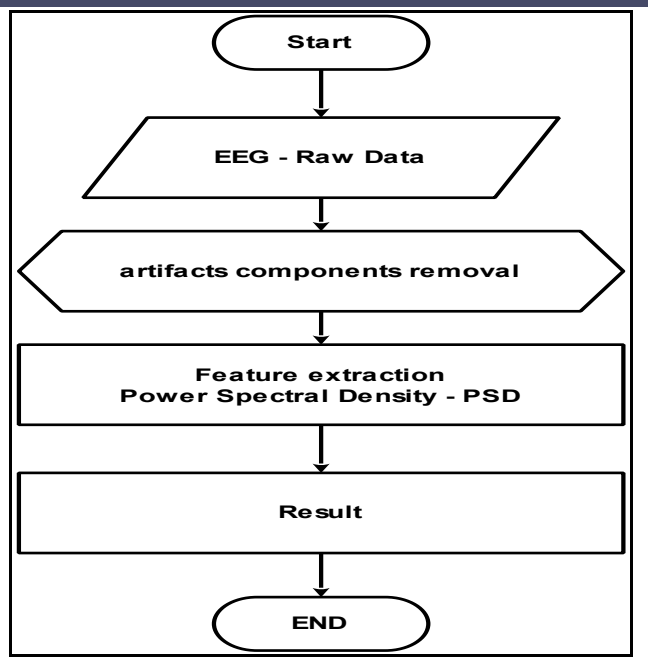

Figure 4. EEG Signal Processing

The mean of PSD was estimated for two channels after combining them. The frequency bands for this study are the whole EEG signal, theta band, alpha band, and the beta band as explained in signal characteristics Table 1. The PSD for each band is illustrated in Figure 8.

To obtain whole EEG power calculation, it was necessary to gain the total power of the band from 1 to $42 \mathrm{~Hz}$, while Theta\Alpha Ratio (TAR) and ThetalBeta Ratio (TBR) estimation by using the equation (8); where P (SB) is the power of slow band such as theta, and $\mathrm{P}(\mathrm{FB})$ is a power of fast bands such as alpha and beta.

$$
\text { Ratio }=\frac{\mathbf{P}(\mathbf{S B})}{\mathbf{P}(\mathbf{F B})}
$$

The logarithmic unit decibels $(\mathrm{dB})$ used for measuring the average power spectral density that is easy to view small components in each frequency band, mean of power is average spectrum over segments for each frequency band to the total of the spectrum. As explained in the $\mathrm{Y}$-axis of Figure 8, the out-PSD plot is power $(\mathrm{dB}) / \mathrm{Hz}$ to bandwidth in the $\mathrm{x}$-axis.

Table 1: Signal Characteristics

\begin{tabular}{cc}
\hline \hline Signal characteristics & Details \\
\hline & Pre and post sessions: 5 (Minutes) \\
\cline { 2 - 2 } Time period & During stimulation session: 17 (Minutes) \\
Number of channels & 2 \\
Sampling frequency & $256(\mathrm{~Hz})$ \\
Frequency bands & \\
Theta band & $4: 0.5: 7$ (Hertz) \\
Alpha band & $8: 0.5: 12$ (Hertz) \\
Beta band & $13: 0.5: 30$ (Hertz) \\
Whole EEG band & $1: 0.5: 42$ (Hertz) \\
\hline \hline
\end{tabular}




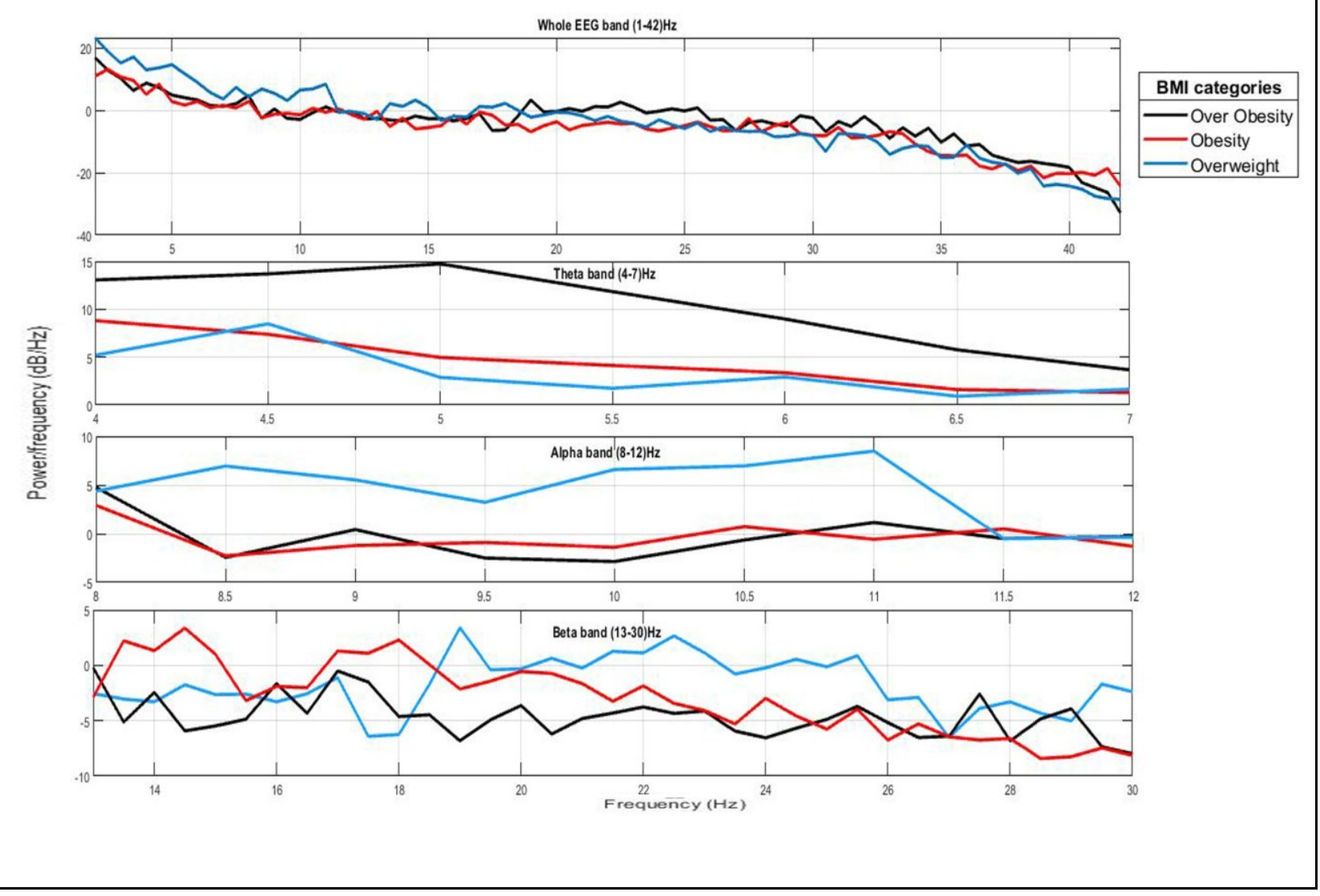

\section{2) Statistical analysis}

Figure 5. Power Spectral Density of EEG Bands

The statistical analysis started after the extraction of features from both data types: the self-report questionnaires and EEG signals for all participants' groups. The data were organized and inserted into a datasheet to assess the values by SPSS. Descriptive analysis was applied to find out the participants' characteristics, namely the mean BMI of participants, gender, head-size (manually calculated). Also, the same model was applied to find out the mean of quantitative data that was related with PSD of EEG features and the median of qualitative data that was related with self-report questionnaires. The two-way repeated measurement ANOVA model was applied to study the comparison between the mean of EEG features between participants' group at the study phases. Also, the comparison of the median of the questionnaire terms between participants group at pre and post stimulation phases. The P-value and z-value are computed to evaluate the significant level in data variances. Then, the spearman correlation was applied to estimate the relationship between the variance in EEG features and variance in questionnaire terms.

\section{Result and Discussion}

Twenty-four adults (16 males and 8 females; age ranged between 41-18) were recruited from excess weight individuals (overweight, obesity, and obesity). Their characteristics are summarized in Table 2. These details showed that all participants recruited for the study met the inclusion criteria which proves the reliability of results. Participants were randomly assigned 
to either Experimental Group (EX) which included EEG-NF experiment, and Control Group (C) without experiment. Also, the head-size for all participants was recorded and the mean of length between nasion-inion is $38.41(2.61) \mathrm{cm}$ while the mean of head-circumference is $54.5(5.04) \mathrm{cm}$. These values referring to the importance of identifying the EEG electrodes' location on the prefrontal cortex.

In summary, this section reports that all participants from excess weight individuals are assigned into groups randomly so that any differences that appear in the post phase analysis should be the result of the experiment effect, this has good internal validity of results.

Table 2: Participants Characteristics



\section{1) Data Analysis on Pre-Phase Stimulation at Two Groups}

\section{Mean Variance of EEG Parameters}

The descriptive analysis was used to find the mean value of EEG power for each group. For the EX-group is (59.98), the confidence intervals range between (55.60-64.35), while the mean value in C-Group is (56.89) and the confidence intervals range between (53.28-60.49). Regarding TBR as shown in table 4.2, the mean value of TBR is (2.30) in Ex-Group with confidence intervals range is (2.07-2.54), while the mean value is (2.51) with confidence intervals range (2.25-2.76) in $\mathrm{C}$ group. Also, the mean value of TAR was (1.50) with confidence intervals range (1.38-1.62) in EX-group, and (1.51) with confidence intervals range (1.37-1.64) in the control group. The p-value for all these parameters $>0.05$ so this indicates a non-significant variance between groups.

Table 3 reports group differences in EEG power bands of PFC at the pre-stimulation phase. The randomization to groups and a non-significant difference in PFC electrical activity between groups at this phase approves that all percipients had fulfilled the inclusion criteria into groups, and the mean of EEG power signal in excess weight individuals is $58.43 \mathrm{~dB} \backslash \mathrm{Hz}(6.36)$. 
Table 3: Mean Variance of EEG Parameters at Pre-Stimulation

\begin{tabular}{cccc}
\hline \hline \multirow{2}{*}{$\begin{array}{c}\text { EEG Parameters } \\
\text { (Unit) }\end{array}$} & EX (SD) & Groups \\
\cline { 2 - 4 } & $59.98(6.88)$ & $56.89(5.67)$ & P-value \\
\hline EEG power \\
(dB\Hertz) & $2.30(0.36)$ & $2.51(0.39)$ & $\mathbf{0 . 2 4 3}$ \\
TBR (NU) & $1.50(0.19)$ & $1.51(0.21)$ & $\mathbf{0 . 2 0 5}$ \\
TAR (NU) & $\mathbf{0 . 9 4 5}$ \\
\hline \hline
\end{tabular}

\section{Median Variance of Questionnaire terms}

Table 4 observed the median value for each term in two study groups with the p-value. The $\mathrm{P}$ values in all terms is more than 0.05 , which indicated a non-significant variance in response between groups at the pre-stimulation phase. The median value referring to negative trends in food intake behaviour into groups. Despite the randomization to groups and the nonsignificant difference in food intake behaviours between groups which confirm that all participants in the same category of dysfunction in general symptoms of food intake behaviours.

\begin{tabular}{cccc}
\hline \multicolumn{4}{c}{ Table 4: Median Variance of questionnaire terms at Pre-Stimulation } \\
\cline { 2 - 4 } Terms & EX (MR) & Croups & PR) \\
\hline FIB & $2(13.29)$ & $2(11.71)$ & 0.527 \\
CA & $3(13)$ & $3(12)$ & 0.660 \\
PWF & $2(12.96)$ & $2(12.04)$ & 0.732 \\
Feeling & $3(14.04)$ & $3(10.96)$ & 0.131 \\
TSE & $2(13.04)$ & $2(11.96)$ & 0.675 \\
Emotion & $2.5(12.92)$ & $2(12.09)$ & 0.754 \\
\hline \hline
\end{tabular}

\section{Correlation Coefficient Between Variables}

Table 5 illustrates the correlation coefficients between the quantitative and qualitative variables of the study at the prestimulation phase. No significant correlations were found between EEG parameters and self-report questionnaire terms except for the whole EEG power with two terms (Food intake, Feelings), despite randomization to groups. The noncorrelation at the pre-stimulation phase between variables is important to prove that there was no difference between the participants prior to the experiment running. This correlation between the whole EEG power and two terms of the questionnaire could be justified as some participant's misunderstanding of these two questions. 
Table 5: Correlation Coefficient at pre-Stimulation

\begin{tabular}{cccc}
\hline \hline $\begin{array}{c}\text { Questionnaire } \\
\text { Terms }\end{array}$ & \multicolumn{3}{c}{ EEG parameters } \\
\cline { 2 - 4 } & $\begin{array}{c}\text { EEG power } \\
(\text { P-value })\end{array}$ & $\begin{array}{c}\text { TBR } \\
\text { ( P-value) }\end{array}$ & $\begin{array}{c}\text { TAR } \\
(\text { P-value })\end{array}$ \\
\hline FIB & $\mathbf{0 . 4 8}(\mathbf{0 . 0 1})$ & $-0.14(0.51)$ & $-0.14(0.50)$ \\
CA & $0.13(0.51)$ & $0.09(0.64)$ & $0.03(0.87)$ \\
PWF & $0.24(0.25)$ & $0.09(0.65)$ & $0.30(0.14)$ \\
Feeling & $\mathbf{0 . 5 5 ( 0 . 0 0 )}$ & $0.16(0.43)$ & $-0.01(0.94)$ \\
TSE & $0.17(0.40)$ & $-0.03(0.86)$ & $-0.06(0.76)$ \\
Emotion & $0.20(0.34)$ & $0.05(0.81)$ & $-0.15(0.47)$ \\
\hline \hline
\end{tabular}

More Significant level P-value $<0.01$. Significant level P-value $<0.05$

\section{2) Data Analysis on Post-Phase Stimulation at Two Groups}

\section{Mean Variance of EEG Parameters}

The first set of analysis at the post-phase examined the impact of stimulation on PFC activity, therefore, 24 signals were rerecorded for all participants after one month from the pre-phase. The experimental group, throughout this month, was applied to the neurofeedback sessions while the control group was on the waiting list. The post-phase session has the same terms of the pre-phase in EEG signals recording. The mean values with confidence interval range of EEG parameters into groups were computed as shown in Table 6 .

The mean values of EEG power in EX-group (47.44) with confidence interval range (43.06 -51.82), and the mean value in C-Group was (57.68) with confidence interval range (54.08-61.28) and $\mathrm{P}<0.01$, the result indicates that there is a significant variance at the whole EEG power into groups.

Table 6: Mean Variance of EEG Parameters at post-Stimulation

\begin{tabular}{cccc}
\hline \hline \multirow{2}{*}{$\begin{array}{c}\text { EEG Parameters } \\
\text { (Unit) }\end{array}$} & EX (SD) & Groups & P-value \\
\cline { 2 - 4 } & $47.44(6.88)$ & $57.68(5.66)$ & $\mathbf{0 . 0 0 1}$ \\
EEG power & $1.84(0.33)$ & $2.55(0.40)$ & $\mathbf{0 . 0 0 0}$ \\
TBR $\backslash$ Hertz) & $1.40(0.17)$ & $1.53(0.21)$ & $\mathbf{0 . 3 1 1}$ \\
TAR (NU) & $1.45($ Mor $)$
\end{tabular}

Also, the mean value of TBR was (1.84) in the Ex-Group and confidence interval range (1.62-2.06) while (2.55) with interval range (2.29-2.80) in C-group. The p-value $<0.01$ that means there was a very significant variance at TBR into groups. Regarding TAR, the mean value was (1.42) in EX-group and (1.32) in the control group with confidence interval range (1.33-1.56) in EX-group and (1.39-1.66) in C-group. The P-value $>0.05$, these values indicate that there was a nonsignificant variance in TAR between groups. 
Table 6 reports group differences in EEG power bands at the post-stimulation phase. The significant variance in the whole EEG power and TBR into groups at $\mathrm{P}<0.01$, while there was a non-significant variance on TAR. This variance justified by the impact of the EEG-NF experiment at EX-group because they have completed 8 sessions of the NF stimulation phase while the control group was on the waiting list. The TBR significant variance into groups was due to the neurofeedback protocol.

It seems that the Beta band was the desired EEG bands more than the alpha band during the cue-exposure task, that approves a Beta band of PFC electrical activity is the fast EEG bands that is related to environmental factors of food intake behaviours more than the Alpha band.

\section{Median Variance of Questionnaire terms}

Table 7 illustrates the variance in the questionnaire terms into groups, all terms have a significant variance while only the feeling term showed a non-significant level. The source of this vibration was justified by the effect of the EEG-NF experiment to change the food intake behaviours in EX-group. The more surprising correlation is with the EX-group which has changed their responses in a positive tendency towards the questionnaire while the control group did not have any significant change. In summary, the positive tendency of the questionnaire indicates an improvement in food intake behaviours. These results are following targets of PFC stimulation, even though the NF used in previous studies were mostly with cognitive behaviours improvement or mental disorders treatments such as ADHD or addiction [20], [21] or some eating disorder such as "restrained eaters" [15], these result showed similar treatment trends but in improving general symptoms of food intake behaviour in obesity and overweight cases.

Table 7: Median Variance of questionnaire terms at post-Stimulation

\begin{tabular}{cccc}
\hline \hline & \multicolumn{3}{c}{ Groups } \\
\cline { 2 - 4 } Terms & EX (MR) & C (MR) & P-value \\
\hline FIB & $1(8.33)$ & $2(16.67)$ & 0.002 \\
CA & $1(9.33)$ & $2(15.67)$ & 0.016 \\
PWF & $1(8.17)$ & $2(16.83)$ & 0.000 \\
Feeling & $1(10.04)$ & $2(14.96)$ & 0.076 \\
TSE & $1(8.50)$ & $2(16.50)$ & 0.001 \\
Emotion & $1(9.12)$ & $2(15.99)$ & 0.010 \\
\hline \hline
\end{tabular}

More Significant level P-value $<0.01$. Significant level P-value $<0.05$., MR: Mean rank 


\section{Correlation Coefficient Between Variables}

Table 8 shows the result of the correlational analysis between two data types at the post-stimulation phase. The positive correlation was found between the whole EEG power and all terms of the questionnaire at a significant level. There was a positive correlation between TBR and FIB, CA, and PWF terms at a significant level, also, positive correlation with other terms at a non-significant level. These results were collected at the post stimulations phase, so this correlation could be justified by the effect of EEG-experiment on the electrical activity of PFC of EX group which led to changes in general symptoms of food intake behaviour of the participants according to their self-report.

The questionnaire answers changed to a positive trend in improving the food intake behaviour. This change was because of the decrease that took place in the EEG power in general, and in the theta and beta ratio in particular. While, no significant correlation was found between TAR and the questionnaire terms as shown in the below table, due to the EEG-experiment hadn't affected on an alpha band of PFC.

Table 8: Correlation Coefficient at Post-Stimulation

\begin{tabular}{|c|c|c|c|}
\hline \multirow{2}{*}{$\begin{array}{c}\text { Questionnaire } \\
\text { Terms }\end{array}$} & \multicolumn{3}{|c|}{ EEG parameters } \\
\hline & $\begin{array}{c}\text { EEG power } \\
\text { (P-value) }\end{array}$ & $\begin{array}{c}\text { TBR } \\
\text { ( P-value) }\end{array}$ & $\begin{array}{c}\text { TAR } \\
\text { ( P-value) }\end{array}$ \\
\hline FIB & $0.48(0.01)$ & $0.41(0.04)$ & $-0.05(0.80)$ \\
\hline CA & $0.51(0.01)$ & $0.48(0.01)$ & $0.14(0.49)$ \\
\hline PWF & $0.54(0.00)$ & $0.48(0.01)$ & $0.28(0.18)$ \\
\hline Feeling & $0.47(0.01)$ & $0.30(0.14)$ & $0.21(0.32)$ \\
\hline TSE & $0.51(0.01)$ & $0.36(0.08)$ & $0.15(0.45)$ \\
\hline Emotion & $0.49(0.01)$ & $0.14(0.51)$ & $\begin{array}{ll}0.01 & 0.95)\end{array}$ \\
\hline
\end{tabular}

\section{3) Comparison between Data on Pre and Post Stimulation Phases}

The above section reports the significant variance in the outcomes which are quantitative variables (EEG parameters) and qualitative variables (self-report questionnaires), wherefore, it is necessary here to clarify exactly what the sources of these variables' variation are. This study designed two groups, random selection, and pre and post phases, so the sources of variables variation calculated was based on statistics coefficients for phases, groups, and interaction between phases and groups.

The next sections discuss the coefficients df, F, Mean Square, P-value, and Partial Eta Squared for these sources with study variables and according to these values, we could evaluate the effect size of these sources on experiment variables variation. The high effect size of sources indicates an increase in coefficients values and a decrease when modest effect size. The pvalue evaluates the significant level of effect size. 
A two-way repeated measure ANOVA was applied to assess whether there was a difference in the respondents' EEG signals between groups and phases as shown in Table 9. The findings for the within phase effect of repeated measures ANOVA was significant $(\mathrm{F}=706.88 . \mathrm{p}=0.000, \mathrm{~h} 2=0.970)$. These results indicate that the interaction between the group and the phase was statistically significant $(\mathrm{F}=910.09 . \mathrm{p}=0.000, \mathrm{~h} 2=0.976)$ indicated that the changes of the whole EEG power in both groups were significantly different across times (pre and post-stimulation phase). The main effect of the group was not statistically significant $(\mathrm{F}=1.94, \mathrm{p}=0.177, \mathrm{~h} 2=0.081)$.

These results may be explained by the fact that within phase changes, the whole EEG power varied depending on phase and phase*group and in a way strikingly different from the effects observed for the whole EEG power, which was confirmed by the significant variance in the whole EEG power between phases on EX-group and no significant difference between phases on C-group as illustrated in the previous section. To test the related hypothesis, the post hoc test (Bonferroni) was applied to compare the mean scores.

According to the result of the Bonferroni test that is shown in Table 9, the difference of the whole EEG power means score between pre-phase and post-phase in the experimental group was statistically different $(\mathrm{p}<0.01)$ while in both pre-phase and post-phase there was a significant difference between the control group $\mathrm{p}<0.05$. The table below indicates the mean difference and h2 of EEG power in the Ex-group more than the control group that interpreted the effect of stimulation sessions on the whole EEG power in the EX-group.

Table 9: Difference between Phases at Two Groups

\begin{tabular}{|c|c|c|c|c|c|}
\hline \multirow{2}{*}{ Source of variation } & \multicolumn{5}{|c|}{ ANOVA in Whole EEG Power } \\
\hline & df & MS & $\mathrm{F}$ & P-value & $\eta 2$ \\
\hline Phase & 1 & 413.83 & 706.88 & 0.000 & 0.970 \\
\hline Phase * Group & 1 & 532.8 & 910.09 & 0.000 & 0.976 \\
\hline Group & 1 & 153.29 & 1.94 & 0.177 & 0.081 \\
\hline \multirow[b]{2}{*}{ Group } & \multicolumn{5}{|c|}{ Comparison between Pre-post Stimulation Phases } \\
\hline & $\mathrm{N}$ & $\Delta \mu$ & $\mathrm{SD}$ & P-value & $\eta 2$ \\
\hline Control & 12 & -0.023 & 0.007 & 0.002 & 0.354 \\
\hline Experimental & 12 & 0.053 & 0.007 & 0.000 & 0.741 \\
\hline
\end{tabular}

Based on questionnaires scores, the acceptable hypothesis is $H 1: \mu 1>\mu 2$, where $\mu 1$ is the median of self-report questionnaire terms in pre-phase and $\mu 2$ is the median of self-report questionnaire terms in post-phase in EX-group. The null hypothesis is rejected. Referring to Table 10, the median of questionnaire terms in $(\mu 1)$ for EX-group is greater than ( $\mu 2)$, these results indicate that EEG-NF experiment has affected food intake behaviour of the participants by manipulating the PFC electrical activity, while no differences in the median of terms into the control group. 
The comparison between the outcomes of the questionnaire in the pre and post-stimulation phase referred to a positive change in participants' responses of EX-Group which is related to an improvement in food intake behaviour such as craving action, trouble eating stopping, and emotions based on response scores. That indicates that the NF stimulation trial has an impact on EX-groups behaviour. While the control group non-significant change in responses that because of no PFC stimulation.

In summary, these results illustrated the significant change in median values of terms at EX-group into a positive tendency that indicates the effect of PFC stimulation by EEG-NF on improving food-intake behaviour.

\begin{tabular}{|c|c|c|c|c|c|}
\hline Terms & Phase & Group & $\mathbf{N}$ & Median & SD \\
\hline \multirow{4}{*}{ FIB } & Pre-phase & EX-Group & 12 & 2 & 0.51 \\
\hline & Pre-phase & C-Group & 12 & 2 & 0.62 \\
\hline & Post-Phase & EX-Group & 12 & 1 & 0.49 \\
\hline & Post-Phase & C-Group & 12 & 2 & 0.62 \\
\hline \multirow{4}{*}{$\mathbf{C A}$} & Pre-phase & EX-Group & 12 & 3 & 0.45 \\
\hline & Pre-phase & C-Group & 12 & 3 & 0.49 \\
\hline & Post-Phase & EX-Group & 12 & 1 & 0.67 \\
\hline & Post-Phase & C-Group & 12 & 2 & 0.62 \\
\hline \multirow{4}{*}{ PWF } & Pre-phase & EX-Group & 12 & 2 & 0.83 \\
\hline & Pre-phase & C-Group & 12 & 2 & 0.66 \\
\hline & Post-Phase & EX-Group & 12 & 1 & 0.38 \\
\hline & Post-Phase & C-Group & 12 & 2 & 0.71 \\
\hline \multirow{4}{*}{ Feeling } & Pre-phase & EX-Group & 12 & 3 & 0.28 \\
\hline & Pre-phase & C-Group & 12 & 3 & 0.66 \\
\hline & Post-Phase & EX-Group & 12 & 1 & 0.88 \\
\hline & Post-Phase & C-Group & 12 & 2 & 0.62 \\
\hline \multirow{4}{*}{ TSE } & Pre-phase & EX-Group & 12 & 2 & 0.75 \\
\hline & Pre-phase & C-Group & 12 & 2 & 0.57 \\
\hline & Post-Phase & EX-Group & 12 & 1 & 0.45 \\
\hline & Post-Phase & C-Group & 12 & 2 & 0.71 \\
\hline \multirow{4}{*}{ Emotion } & Pre-phase & EX-Group & 12 & 2.5 & 0.77 \\
\hline & Pre-phase & C-Group & 12 & 2 & 0.75 \\
\hline & Post-Phase & EX-Group & 12 & 2 & 0.51 \\
\hline & Post-Phase & C-Group & 12 & 2 & 0.62 \\
\hline
\end{tabular}

N: Number of Participants, SD: standard deviation

\section{Difference Value between EEG Parameters at Two Phases}

As explained in previous sections, there is no significant variance in the mean values of EEG parameters $(\mu E E G, \mu T B R$, $\mu \mathrm{TAR}$ ) between groups at the pre-phase because the data were collected before the NF stimulation phase, while there is a significant variance in the mean values of the whole EEG power ( $\mu \mathrm{EEG)}$ and TBR ( $\mu \mathrm{TBR}$ ) between groups at the post phase. That means, after NF stimulation phase was applied on EX-group, so this variance could be justified by the NF 
stimulation protocol especially when the protocol focused on theta and beta bands more than the alpha band of PFC electrical activity, therefore, there is no significant variance in ( $\mu$ TAR) between groups at the same phase. Table 11 illustrates the mean difference between the mean of EEG features at both pre and post phases by subtracting the mean values of EEG feature in the pre-phase from the mean values of EEG features in the post phase to calculate the mean difference between them, this computation is needed to evaluate the impact of EEG-NF stimulation on EEG signals of the prefrontal cortex.

The mean of a difference value between ( $\mu$ EEG1 - $\mu$ EEG2) for 12 participants in EX-group is 12.53 with interval range (11.61-13.45), while the mean of difference values in C-group was -0.79, the mean values in C-control referred to the nonsignificant difference in EEG power feature in C-group, the reason of this variance is the EEG-NF stimulation sessions that used on EX-group and while the C-group do not have these stimulation sessions.

This table illustrates the difference of TBR means ( $\mu$ TBR1 - $\mu$ TBR2) on EX-group which was 0.46 , while in C group was 0.04 and the mean values in 24 participants were 0.21 , the P-value was $<0.01$. Regarding the TAR features, the mean values of the difference between the two phases are 0.05 in EX-group and - 0.02 in C-group and the mean value of the total participants is 0.01 with $\mathrm{P}$-value $<0.01$. These data are used to find the correlation between different values of EEG parameters.

Table 11: difference between Phases at Two Groups

\begin{tabular}{cccc}
\hline \hline & \multicolumn{3}{c}{ Groups } \\
\cline { 2 - 4 }$\Delta(\mu$ pre - $\mu$ post $)$ & EX (SD) & C (SD) & P-value \\
\hline EEG1-EEG2 & $\mathbf{1 2 . 5 3 ( 1 . 4 4 )}$ & $-\mathbf{0 . 7 9}(\mathbf{0 . 4 9})$ & $\mathbf{0 . 0 0 0}$ \\
TBR1-TBR2 & $\mathbf{0 . 4 6}(\mathbf{0 . 0 7})$ & $\mathbf{- 0 . 0 4}(\mathbf{0 . 0 5})$ & $\mathbf{0 . 0 0 0}$ \\
TAR1-TAR2 & $\mathbf{0 . 0 5}(\mathbf{0 . 2 6})$ & $\mathbf{- 0 . 0 2}(\mathbf{0 . 0 2})$ & $\mathbf{0 . 0 0 0}$ \\
\hline \hline & More Significant level P-value $<0.01$, Significant level P-value $<0.05$, SD: Standard Deviation &
\end{tabular}

More Significant level P-value $<0.01$, Significant level P-value $<0.05$, SD: Standard Deviation

The correlation analysis used to find the correlation inside and between the EEG features and find the strong band ratio that relates to the whole EEG power. The correlation between EEG power and power bands ratio is interesting to estimate the impact percentage of the ratio bands on the whole EEG signal. The plus values in Table 12 indicates a perfect positive correlation between EEG parameters. The correlational analysis result referred to $96 \%$ strength of correlation between the EEG power and TBR at a significant level. While $87 \%$ with $\mathrm{P}$-value $<0.01$ between EEG power and TAR, regarding the TBR and TAR the value is $81 \%$ with $\mathrm{P}$-value $<0.01$.

These results indicate a more significant correlation between parameters in a positive relationship and the TBR estimated is stronger than the TAR with the whole EEG power. That means the TBR impacts and reflects changes on the whole EEG power more than TAR. 
Table 12: Correlation Coefficient between EEG Parameters

\begin{tabular}{cc|c|c}
\hline \hline$\Delta$ Difference & \multicolumn{3}{c}{ Correlation ( P-value) } \\
\cline { 2 - 4 } & EEG Power & TBR & TAR \\
\hline EEG power & - & $0.96(0.00)$ & $0.87(0.00)$ \\
TBR & $0.96(0.00)$ & - & $0.81(0.00)$ \\
TAR & $0.87(0.00)$ & $0.81(0.00)$ & - \\
\hline \hline
\end{tabular}

\section{Conclusion}

This study focused on studying the feasibility of utilizing an NF device in manipulating the PFC activity which leads to modification in general symptoms of food intake behaviour in excess weight individuals. The analysis of variance in EEG bands of PFC on the pre-stimulation phase between two study groups was statistically non-significant at $\mathrm{P}>0.05$, while the analysis of variance in EEG bands on the post-stimulation phase between two groups was statistically significant at $\mathrm{P}<0.01$ except for the TAR where there was a non-significant variance. The comparison between the mean values for the whole EEG power and TBR leads to the conclusion that the null hypothesis rejected while for TAR the null hypothesis is accepted.

In summary, these results reflect the cue-exposure EEG-NF protocol's impact on whole EEG power, particularly theta and beta bands based on significant TBR variation. This study has supported the effectiveness of the cue-exposure EEG-NF experiment on changing the tendency of general symptoms of food intake behaviour from the negative side to the positive side according to self-report questionnaire responses. The study hypothesis is $\mu 1>\mu 2$, where the $\mu 1$ is the median of selfreport questionnaire terms in pre-phase while the $\mu 2$ is the median of self-report questionnaire terms in post-phase in the EXgroup. Based on the data analysis, the null hypothesis is rejected. The questionnaire terms, except the feeling terms, were statistically significant at $\mathrm{P}<0.05$ between pre and post phases. The reason for that is the stimulation phase sessions had affected the EX-group responses to the food intake behaviour questionnaire. The decrement in the median values indicates an improvement in general symptoms of food intake behaviour based on the design of the questionnaires. Hence, the correlation analysis between quantitative and qualitative variables indicated a positive correlation between decrement in EEG parameters and improvement in food intake behaviour. The whole EEG power and TBR decreased after the stimulation phase and questionnaire terms change the tendency from negative side to positive side, so that it confirmed an EEG-NF experiment had a positive result in the decrement of TBR for PFC activity to modify the general symptoms of food intake behaviour in excess weight individuals.

The proposed study was able to achieve and evaluate its hypothesizes by combining the quantitative and qualitative analysis, the qualitative data represented by self-report questionnaire, and quantitative data drawn by the PSD of EEG signals. Most of 
the previous EEG-NF studies that targeted food intake behaviour or eating disorders depend only on qualitative variables for stimulation evaluation.

The limitations of this study are; firstly, the monitoring of food or calorie intake size and change in body mass index are out of this study parameters, it focused on modifying the food intake behaviour only by depending on evaluating the questionnaire responses. Second, the EEG recording mechanism used two channels only. This mechanism is not costly and easy to use with a significant result to prove the study's hypothesis, but for future work on intelligent system training or classification approach, this mechanism requires and upgrade.

This study provides preliminary support for the therapeutic potential of cue-exposure EEG-NF experiment that targets the prefrontal cortex, to influence neural processes underlying food intake behaviour in overweight and obesity cases. The NF protocol used in this study was weekly sessions that allowed the subjects to stay longer in the stimulation phase. The implementation of an affordable and suitable EEG system was successfully carried out. In summary, the EEG-NF is one of the brain stimulation techniques, a safe, non-surgical, affordable price system, and easy to handle compared with other techniques. There are no negative side effects compared with a surgical interface such as DBS. Also, the electrodes have free skin effect on PFC areas such as redness compared with tDCS or TMS techniques.

\section{Declarations}

This study was approved by the Ethics Committee of Research involving Human Subjects of University Putra Malaysia (JKEUPM), reference number (2017-294), date 02. May.2018.

The consent form had been signed by the participants before the experiment started, permission for publication is included already in the form. The data that support the findings of this study are available from the faculty of engineering - electrical and electronic department in University Putra Malaysia as the third party. Restrictions apply to the availability of these data, which were used under license for the current study, and so are not publicly available. Data are however available from the authors upon reasonable request and with permission of the third party. The authors declare that they have no competing interests This research is financially supported by Putra Grant from University Putra Malaysia.

Authors contributions: Mohammed worked on-call for participants, data collection, analysed and interpreted the data. Asnor, Hafiz, and Siti A. examined the study problem and evaluated the study results and were major contributors in writing the manuscript. Wan Sulaiman contributed to EEG data recordings and questionnaires structuring. All authors read and approved the final manuscript. 


\section{Reference}

[1] "World health Organization," 2018. [Online]. Available:

http://www.who.int/entity/mediacentre/factsheets/fs311/en/. [Accessed: 01-Sep-2018].

[2] T. Huang, T. Marsh, and M. Moodie, "Changing the future of obesity: science, policy, and ... [Lancet. 2011] - PubMed - NCBI," vol. 378, no. 9793, pp. 838-847, 2012.

[3] M. Führer, D., Zysset, S., \& Stumvoll, "Brain Activity in Hunger and Satiety: An Exploratory Visually Stimulated fMRI Study," Obesity, no. 16(5), pp. 945-950, 2008.

[4] M. E. Gluck et al., "Neuromodulation Targeted to the Prefrontal Cortex Induces Changes in Energy Intake and Weight Loss in Obesity," Obes. J. Symp., vol. 23, no. 11, pp. 2149-2156, 2015.

[5] M. E. Gluck, P. Viswanath, and E. J. Stinson, "Obesity, Appetite, and the Prefrontal Cortex," Curr. Obes. Rep., vol. 6, no. 4, pp. 380-388, 2017.

[6] C. J. Lowe, C. Vincent, and P. A. Hall, "Effects of Noninvasive Brain Stimulation on Food Cravings and Consumption: A Meta-Analytic Review," Psychosom. Med., vol. 79, no. 1, pp. 2-13, 2017.

[7] I. C. Macedo et al., "Repeated transcranial direct current stimulation reduces food craving in Wistar rats," Appetite, vol. 103, pp. 29-37, 2016.

[8] S. Mostafavi, A. Khaleghi, and M. R. Mohammadi, "Is transcranial direct current stimulation an effective modality in reducing food craving? A systematic review and meta-analysis Is transcranial direct current stimulation an effective modality in reducing food craving? A systematic review and meta-anal," Nutr. Neurosci., vol. 0, no. 0, pp. 1-13, 2018.

[9] O. M. Lapenta, K. Di Sierve, E. C. de Macedo, F. Fregni, and P. S. Boggio, "Transcranial direct current stimulation modulates ERP-indexed inhibitory control and reduces food consumption," Appetite, vol. 83, pp. $42-48,2014$.

[10] L. Perronnet, A. Lécuyer, F. Lotte, M. Clerc, and C. Barillot, "Brain Training with Neurofeedback: Foundations and Methods," Brain-Computer Interfaces 1 Found. Methods, pp. 271-292, 2016.

[11] M. S. Spetter et al., "Volitional regulation of brain responses to food stimuli in overweight and obese subjects: A real-time fMRI feedback study," Appetite, vol. 112, pp. 188-195, 2017.

[12] N. Ihssen, M. O. Sokunbi, A. D. Lawrence, N. S. Lawrence, and D. E. J. Linden, "Neurofeedback of visual food cue reactivity: a potential avenue to alter incentive sensitization and craving," Brain Imaging Behav., vol. 11, no. 3, pp. 915-924, 2017.

[13] M. I. Al-hiyali, A. J. Ishak, H. Harun, S. A. Ahmad, and W. S. Wa, "A Review in Modification Food-Intake Behavior by Brain Stimulation : Excess Weight Cases,” Neuroquantology, vol. 16, no. 12, pp. 86-97, 2018.

[14] B. Sathian, J. Sreedharan, S. N. Baboo, and et al, "Relevance of Sample Size Determination in Medical Research,” Nepal J. Epidemiol., vol. 1, no. 1, pp. 4-10, 2010.

[15] J. Schmidt and A. Martin, "Neurofeedback Reduces Overeating Episodes in Female Restrained Eaters: A Randomized Controlled Pilot-Study," Appl. Psychophysiol. Biofeedback, vol. 40, no. 4, pp. 283-295, 2015.

[16] S. Enriquez-Geppert, R. J. Huster, and C. S. Herrmann, "EEG-Neurofeedback as a Tool to Modulate Cognition and Behavior: A Review Tutorial," Front. Hum. Neurosci., vol. 11, no. February, pp. 1-19, 2017.

[17] W. Nan, F. Wan, M. I. Vai, and A. C. Da Rosa, "Resting and Initial Beta Amplitudes Predict Learning Ability in Beta/Theta Ratio Neurofeedback Training in Healthy Young Adults," Front. Hum. Neurosci., vol. 


\section{$9,2015$.}

[18] C. W. E. M. Quaedflieg, F. T. Y. Smulders, T. Meyer, F. Peeters, H. Merckelbach, and T. Smeets, "The validity of individual frontal alpha asymmetry EEG neurofeedback," Soc. Cogn. Affect. Neurosci., vol. 11, no. 1, pp. 33-43, 2015.

[19] A. Delorme and S. Makeig, "EEGLAB : an open source toolbox for analysis of single-trial EEG dynamics including independent component analysis," vol. 134, pp. 9-21, 2004.

[20] A. Feil, Jodie and Zangen, "Brain stimulation in the study and treatment of addiction," Neurosci. $\mid \&$ Biobehav. Rev., vol. 34, no. 4, pp. 559-574, 2010.

[21] Y. Wang et al., "Relative Power of Specific EEG Bands and Their Ratios during Neurofeedback Training in Children with Autism Spectrum Disorder," Front. Hum. Neurosci., vol. 9, no. January, 2016. 